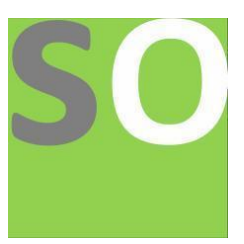

Article title: Mycotoxigenic fungi distribution and Water Quality of raw and treated water from Ede-Erinle and Opa waterworks systems, Osun State Nigeria

Authors: Abayomi Oyewale Tolulope[1], Taiwo Adesakin[2]

Affiliations: Institute of Ecology and Environmental Studies, Faculty of Science, Obafemi Awolowo University, Ile-Ife, Nigeria[1], Department of Biology, Faculty of Life Sciences, Ahmadu Bello University, Zaria, Nigeria[2]

Orcid ids: 0000-0003-3547-0152[2]

Contact e-mail: ataiwonelson@gmail.com

License information: This work has been published open access under Creative Commons Attribution License http://creativecommons.org/licenses/by/4.0/, which permits unrestricted use, distribution, and reproduction in any medium, provided the original work is properly cited. Conditions, terms of use and publishing policy can be found at https://www.scienceopen.com/.

Preprint statement: This article is a preprint and has not been peer-reviewed, under consideration and submitted to ScienceOpen Preprints for open peer review.

DOI: 10.14293/S2199-1006.1.SOR-.PP9NKCJ.v1

Preprint first posted online: 05 November 2020

Keywords: Mycotoxigenic fungal, health risk, treatment, nutrients, environmental variables 


\title{
Mycotoxigenic fungi distribution and Water Quality of raw and treated water from Ede-Erinle and Opa waterworks systems, Osun State Nigeria
}

\author{
Abayomi Tolulope Oyewale ${ }^{1}$ and Taiwo Adekanmi Adesakin ${ }^{2}$ \\ ${ }^{1}$ Institute of Ecology and Environmental Studies, Faculty of Science, Obafemi Awolowo \\ University, Ile-Ife, Nigeria \\ ${ }^{2}$ Department of Biology, Faculty of Life Sciences, Ahmadu Bello University, Zaria, Nigeria
}

\section{Corresponding Author:}

Taiwo Adekanmi Adesakin

Email: ataiwonelson@gmail.com

\begin{abstract}
This present study seeks to investigate the impact of environmental variables on the mycotoxigenic fungi distribution in different stages of water treatment from Ede-Erinle and Opa water work systems. The sampling stations were grouped into raw, treated and distributed stations for each of the waterworks system and samples were collected on bi-monthly basis for one annual cycle, spanning through the rainy and dry seasons. A total number of 30 fungal species which belonged to 7 classes were isolated from both Ede and Opa water works systems during the study. This included 23 species from Ede water works and 14 fungal species from Opa water works system. Ascomycetes contributed $41.6 \%$ out of total fungal classes identified from Ede waterworks system followed by zygomycetes $(33.3 \%)$, Deuteromycete $(12.5 \%)$ while Penicillium and Mucor contributed $20.83 \%$ out fungal genera identified and aspergillus (16.67\%). Zygomycetes accounted for $30.77 \%$ out of total fungal classes isolated from Opa waterworks system followed by ascomycetes (23.05\%), Eusacomycetes and Dothideomycetes contributed $15.38 \%$ while Apsergillus, auerobasidium, rhizopus and cladosporium had the highest percentage contribution of $15.38 \%$ among the fungal genera identified. There was strong correlation between total Heterotrophic fungal distribution and water temperature and $\mathrm{pH}$ in water samples from Ede-Erinle waterworks systems that influenced the growth of mycotoxicin fungal while the nutrient parameters such as nitrate and phosphate influenced the abundance of fungi in water samples from Opa waterworks systems. In Ede-Erinle and Opa waterworks systems, the highest mean values for water temperature and phosphate were observed from distribution water while the mean concentration of $\mathrm{pH}$ and total heterotrophic fungal counts were higher in raw water samples. However, nitrate was higher in the treated water sample during the study. The presences of mycotoxigenic fungal such as Aspergillus sp, Mucor sp, Cladosporium $s p$., Penicillium sp., and Trichoderma sp from the treated and distribution water from Ede and Opa water work system after the water has undergo treatment process could pose serious health risks to consumers because they can cause aspergillosis, mucormycosis and penicilliosis infections respectively in human.
\end{abstract}

Keywords: Mycotoxigenic fungal, health risk, treatment, nutrients and environmental variable 


\section{INTRODUCTION}

Clean water that is fit for human use makes up just $0.6 \%$ of the global water supplies contained in glaciers, surface water and groundwater (Wurzbacher et al., 2011). Depending on the geological characteristics of the area, either groundwater or surface water is the primary source for the production of tap water (DEFRA, 2011; Gray, 2014). The rainwater is also a relevant source in other regions of the world. The presence, colonization and growth of fungi in tap water therefore depends on a variety of factors, such as location of the primary source of water, sunlight, temperature, ion and $\mathrm{pH}$ composition, presence of organic material, concentration of dissolved oxygen, water treatment, use of materials for water delivery systems as well as the possibility of biofilm formation. Generally, most water systems treat water through coagulation, flocculation, sedimentation and filtration, which are intended to remove microbial contaminants by the application of disinfection, such as chlorine, as an integral component of water treatment (Adam et al. 1998). The literature has shown that water treatment plant processes do not fully kill all pathogenic microorganisms, including fungi in water, which end up at the point of use in treated water delivery systems (Sammon et al. 2011; Pereira et al. 2013).

Fungi are natural inhabitants of composting plants, soil and water (Korzeniewska 2011; CalvoPolanco et al. 2016). Terrestrial fungi are capable of migrating from soil into fresh water systems through animals, plants and soil (Wurzbacher et al. 1998; Magwaza et al. 2017). Fungal species such as Fusarium and Aspergillus have been reported for proliferation in water reservoirs where they are involved in waterborne infections (Kanzler et al. 2007). Owing to taste and odor issues, fungi in treated drinking water were first noticed in the 1960s and 1970s, but rarely regarded as not the main subject of the study and also, because the contributory connection between the occurrence of fungi and water quality was not yet understood (Doggett 2000; Hageskal et al. 
2009). The ingestion of fungi-polluted drinking water was not associated with serious disease injuries until research in the 1980s reported a number of cases involving fungal-polluted drinking water (Hageskal et al. 2009). The fungal deposition in water distribution systems is attributed to spores rather than hyphae growth, which raises concerns that the production of mycotoxins' taste and odor problems suggests vegetative growth in situ (Doggett, 2000). The accumulation of microorganisms stored during the water flow is the primary site for the emergence of mouldrelated fungi in the water delivery system; including pathogenic species that are harmful to humans (Grabińska-Łoniewska et al. 2007). Most countries in the world including Nigeria have detected fungi in processed drinking water. Fungi in treated drinking water from the various reports showed that the most prevalent fungi are Acremonium sp., Aspergillus sp., Cladosporium sp., Fusarium sp., Mucor sp., Paecilomyces sp., Penicillium sp., Rhizopus sp., Trichoderma sp. and Verticillium sp. Most of the fungal genera described in the preceding studies are dematiac fungi that can secrete melanin or melanin-like pigment in their cell walls. This makes them thickwalled organisms with hydrophobic spores that give them the advantage of water treatment resistance (Sonigo et al. 2011; Auwal and Taura 2013; Al-gabr et al. 2014; Babič et al. 2017). These persistent fungi normally originate from soil, wood and decomposing plant material (Fox et al. 2016), which explains why they end up in raw water. The species such as Cladosporium sp., Penicillium sp., Fusarium sp., Penicillium sp., Aspergillus sp.,Trichodermasp., Acremonium sp.,and Phialophora sp. can produce mycotoxins and other secondary metabolites producing toxic chemicals that affect water quality and endanger humans and animals (Pitt et al. 2000; Pereira et al. 2010; Sonigo et al. 2011).

Mycotoxins are fungal poisons that are produced by the mycelial structure of filamentous fungi and spores as secondary metabolites (Gupta et al. 2015). Not all fungi produce mycotoxins 
because most of the organisms that produce mycotoxin are filamentous ascomycetes, basidiomycetes and Deuteromycetes, with Penicillium, Aspergillus and Fusarium being the most mycotoxin-producing genera (Abbott, 2002). These mycotoxins have no biochemical effects for fungal growth and its production (Hussein and Brasel, 2001). Fungal growth and mycotoxin development derive from an association between the fungus, the host and the environment (Atanda et al, 2011). The proper combination of these factors determines the amount of colonization of substrates, the shape and the amount of mycotoxin produced (Pitt et al. 2000). The synthesis of any given mycotoxin is not only based on the species but also on the strain (Pitt et al. 2000). Although mycotoxin's chemical structures vary materially, they are usually low organic molecular mass compounds. Because mycotoxins are minute molecules and relatively stable, they are extremely problematic to remove, making it easy for them to join the food and feeding chain while retaining their toxic properties (Omar, 2013). Mycotoxins may be consumed by food or water containing poisonous fungi (Volk, 2013) or taken as mycotoxins which are secreted by fungi without consuming the fungus. If swallowed, mycotoxins induce tissue death, angioinvasion, thrombosis, infarction and other symptoms of the mycosal (Campbell et al, 2004). Mycotoxigenic fungi have been documented in treated drinking water (Paterson and Lima 2008; Al-gabr et al. 2014). While mycotoxin concentrations may be low in water due to dilution, water accumulation in storage tanks, water traveling long distances in distribution systems, the degradation of chlorine residual and the tolerance of some of the fungi to disinfection may contribute to increased concentrations of mycotoxin in drinking water to unacceptable levels (Barriga et al. 2012). Parameters such as temperature and $\mathrm{pH}$ also improve fungal growth and survival in water (Hussain et al, 2011). Small amounts of frequently absorbed mycotoxins could be harmful to human health (Hageskal et al, 2009). 
A variety of infectious, gastrointestinal, skin and life-threatening meningitis infections have included fungi as a causative organism (Pfaller and Diekema 2004; Sulaiman et al. 2014). Alternaria sp., Cladosporium sp., Aspergillus sp., Penicillium sp. and Fusarium sp. have been attributed to allergies and respiratory illness (Korzeniewska 2011; Máiz et al. 2018). Cryptococcus and Candida cause meningitis (Black and Baden 2007), with the Candida species responsible for cutaneous infections (Khan et al. 2010; Volk 2013). Taste and odour problems in water are caused by Aspergillus sp., Acremonium sp., Phialophora sp. and Penicillium sp. (Hageskal et al. 2006; Sonigo et al. 2011). Fungi including Rhizopus, Fusarium, Alternaria, Aspergillus and Penicillium develop mycotoxins that are detrimental to public health because these mycotoxins are cancerous and can weaken the immune system (Bhat et al. 2010; Sonigoet al. 2011; Magwazaet al. 2017). Mycotoxins of major public health concern include aflatoxins (AF), ochratoxins (OT), trichothecenes, zearalenone (ZEN), fumonisins (F), tremorgenic toxins, and alkaloids from ergot (Zain 2011). The types of infections caused by mycotoxigenic pillows depend on the source of mycotoxin, the concentration and period of the exposure, as well as the age, health and sex of the exposed person (Bennett and Klich 2003). Mycotoxins present in water may be extremely diluted and may not be of great concern, but their concentrations may increase leading to dangerous levels of human health, especially if water is stored in reservoirs for longer periods of time (Siqueira, 2011). The lack of toxigenic fungi in processed drinking water does not mean that the water is free of mycotoxins, as mycotoxins may survive long after the death of the fungi (Pitt et al. 2000). Mycotoxins have severe and permanent effects on humans and livestock, as they are believed to be carcinogenic, cytotoxic, mutagenic and may contribute to immunosuppressive complexes (Arroyo-Manzanares et al. 2015). However, the health impacts 
of fungi in treated water are still not well documented and the incidence of outbreaks of waterrelated diseases by pathogenic microorganisms by drinking water has been worrying worldwide.

The purity of water depends on its source, treatment received, storage facilities available and it has greater needs for water treatment to meet it demands for safe drinking water. This present study seeks to investigate how effectiveness of raw water treatment in Ede and Opa water works system and provide information on mycotoxiogenic fungi distribution and their health risk on the water consumption.

\section{MATERIALS AND METHODS}

\section{The Study Area}

The study site were Ede-Erinle and Opa water work systems which serve as the major sources of water supply to twelve Local Government Area in Osun State and the Obafemi Awolowo University Community, respectively. The study was carried out on the raw water sources from the two reservoirs, the treatment stages and their distribution channels. This was with a view to ascertain the quality of water prior to treatment and after it has undergone treatment at the treatment plants to check for changes in quality if any. The study area lie within latitude $07^{\circ} 29^{\prime} 28^{\prime \prime}$ and $07^{\circ} 45^{\prime} 37^{\prime \prime}$ and longitude $004^{\circ} 31^{\prime} 41^{\prime \prime}$ and $004^{\circ} 27^{\prime} 9^{\prime \prime}$ respectively. Egborge (1971, 1981); Lowenberg and Kunzel (1992) reported that the average annual temperature in Ede-Erinle is $26.3{ }^{\circ} \mathrm{C}$ while the average rainfall is $1337 \mathrm{~mm}$. However, the mean annual precipitation of IleIfe is about $1474 \mathrm{~mm}$ with an annual mean temperature of $27.3{ }^{\circ} \mathrm{C}$. The mean annual relative humidity in the study area is about $81.34 \pm 6.73 \%$. The reservoir basins are underlain by granites, undifferentiated schist-gneisses, pegmatilised schists, pegmatites and epidiorite (Ayodele, 1979). The soil of the area are moderately leached, have a low to medium humus 
contents, weakly acidic to neutral topsoil and moderately to strongly acidic sub-soils (Smith and Montgonery, 1962). The study area experience two seasonal periods namely dry and rainy season. The dry season lasted for about four months while the rainy season lasted around eight month to complete an annualcycle. The rainy season is marked by two peak periods, in June/July and September/October. These two periods are separatedby a short dryspell in August. The beginning and end of the rainy season are usually marked by high intensity thunderstorms (Ogunkoya, 2012).

\section{Sampling design and sample Collection}

A total of sixteen sampling stations; eight sampling stations each for Ede-Erinle and Opa waterworks systems was established during the study. The sampling stations were grouped into raw, treated and distributed stations for each of the waterworks system. Samplings were carried out on a bi-monthly basis from October 2017 to August 2018, spanning through the rainy and dry seasons for one annual cycle. Water samples were collected into sterile one litre sampling bottles and 2 litre sterile plastic bottles. Microbiological samples were transported to the laboratory in packed icebox prior analysis.

\section{Enumeration and Isolation of Total Heterotrophic Fungi}

Hundred folds serial dilution of each water sample was carried out respectively six times, in a set of test-tubes, each containing $9.9 \mathrm{ml}$ sterile distilled water. After serial dilution, $1 \mathrm{ml}$ of $10^{2}$ to $10^{5}$ dilutions were aseptically, pipetted into labeled sterile petri dishes. Sterile Potato Dextrose Agar (PDA) of around $45^{\circ} \mathrm{C}$ was poured into each plate and allowed to solidify. The plates were inverted and incubated at $25^{\circ} \mathrm{C}$ for $72 \mathrm{~h}$, after which the plates were checked for growth. After incubation, colonies of different shapes and colours were observed on the plates and their growth 
characteristics (texture, pigmentation, form, spore formation etc) were studied macroscopically. The selected colonies were sub-cultured on fresh PDA plates by carefully cutting on the plate using a sterile inoculating pin. For each set of cut made, the inoculating pin was flamed so that distinct colonies of the organisms were obtained. The plates were incubated at $25{ }^{\circ} \mathrm{C}$ for $72 \mathrm{~h}$. Sub-culturing was repeated until a pure isolate was obtained. Pure culture of different colony types on each plate was obtained and maintained. Once pure isolates are obtained, each isolate was aseptically transferred unto PDA slants and then placed in an incubator for $72 \mathrm{~h}$. Thereafter, they were kept in the refrigerator at $4{ }^{\circ} \mathrm{C}$.

The technique of James and Natalie (2001) was adopted for the morphological identification of the unknown isolated fungi using lactophenol-in-cotton blue stain. The identification was achieved by placing two drops of stain on a clean labeled microscopic slide with the aid of a mounting needle. A small portion of the mycelium from the fungal cultures was aseptically cut and removed and placed in a drop of lactophenol. The mycelium was evenly spread on the slide with the aid of the needle. A cover slip was gently applied with little pressure to eliminate air bubbles. The slide was then mounted and observed with (x10) and (x40) objective lenses respectively. Drawings of the various structures (i.e. sporangium, sporangiophores, columellae, mycelium, spores, etc) of each isolate were made and compared with the drawings of described commonly encountered moulds. The species were identified in accordance to Batnett and Hunter (1998) and Cheesbrough (2000).

\section{Statistical Analysis}

All statistical analyses were undertaken using the SPSS software package (version 25). Analysis of variance (ANOVA) was used to examine spatial differences in physico-chemical parameters and the Duncan's multiple range tests was used to detect pairwise differences between means of factor levels in 
the case of significant differences. Pearson correlation was used on pooled data from all the three stations were used to investigate relationships between mycotogenic fungi distribution with physico-chemical parameters while Microsoft Excel 2016 was used for producing the bar-chart figures.

\section{Results}

A total number of 30 fungal species was isolated from both Ede-Erinle and Opa water works system belonging to 7 classes while 23 species were observed from Ede-Erinle water works system (Reservoir, treated and distribution) and 14 fungal species were recoreded in Opa water works system (Tables 1 and 2). There are 15 fungal isolate were identify from Ede raw water, treated water (17), distribution (10) while Opa raw water (9), treated (8) and distribution (9). Aspergillus fumigatus, Aureobasidium pullularis, Cladosporium herbarum, Rhizopus japonicas and Trichoderma sp contributed $100 \%$ of the total fungal species isolated from Ede waterworks while Aspergillus fumigatus, Rhizopus japonicas, Rhizopus oryzae and Trichoderma sp account for $100 \%$ of the total fungal species identified from Opa waterworks system. There are 9 genera of fungal species identified from Ede waterworks system includes Aspergillus, Penicillium, Cephalosporium, Rhodotorula, Auerobasidium, Rhizopus, Mucor, Trichoderma and Cladosporium while 9 genera of fungal species isolated from Opa waterworks system such as Aspergillus, Neurospora, Entomophthorate, Auerobasidium, Rhizopus, Mucor, Absidia, Trichoderma and Cladosporium (Table 1). Ascomycetes contributed 41.6\% out of total fungal classes identified from Ede waterworks system followed by zygomycetes (33.3\%) and Deuteromycete $(12.5 \%)$ while penicillium and mucor contributed $20.83 \%$ out fungal genera identified and aspergillus (16.67\%). Zygomycetes accounted for $30.77 \%$ out of total fungal classes isolated from Opa waterworks system followed by ascomycetes (23.05\%), Eusacomycetes and Dothideomycetes contributed 15.38\% while Apsergillus, auerobasidium, rhizopus and cladosporium had the highest percentage contribution of $15.38 \%$ among the fungal 
genera identified. The water temperature observed from Ede waterworks system ranged from 26.5-30.50 ${ }^{\circ} \mathrm{C}$ with highest mean water temperature was recorded from distribution station $\left(28.18 \pm 0.19^{\circ} \mathrm{C}\right)$. The $\mathrm{pH}$ ranged concentration vary widely between $3.80-7.50$ while the highest mean value was recorded from raw water sample (7.02 \pm 0.5$)$ and there was high significant differences $(\mathrm{p}<0.01)$ between the treatment water samples. Nitrate highest mean value was observed from treated water sample $(1.03 \pm 1.09 \mathrm{mg} / \mathrm{L})$ while mean value of phosphate was higher in the distribution station $(0.018 \pm 0.03 \mathrm{mg} / \mathrm{L})$. Highest mean values of DO and THF was recorded from raw water sample.The highest mean values for water temperature, phosphate and DO $\left(27.99 \pm 1.56{ }^{\circ} \mathrm{C}, 0.012 \pm 0.01 \mathrm{mg} / \mathrm{L}, 7.08 \pm 1.93 \mathrm{mg} / \mathrm{L}\right)$ was observed from distribution station while $\mathrm{pH}$ mean concentration $(6.53 \pm 0.39)$ was higher for treated water sample and nitrate, total heterotrophic fungal counts was higher in the raw water sample $(1.71 \pm 1.22 \mathrm{mg} / \mathrm{L}$ and $2267020556 \pm 3408914219 \mathrm{cfu} / \mathrm{ml})$. Among the parameters examined in Opa water works system, water temperature, $\mathrm{pH}$, phosphate and THF were with higher mean values recorded from distribution water samples but nitrate was higher in raw water sample while highest mean concentration of DO was observed from treated water sample and there are very high significant differences $(\mathrm{p}<0.001)$ between treatments (Table 3$)$.

Seasonally, the highest mean value for water temperature was recorded from treated water sample $\left(29.2 \pm 0.78{ }^{\circ} \mathrm{C}\right)$ during dry season while the lowest was observed from distribution water sample $\left(27.61 \pm 0.60{ }^{\circ} \mathrm{C}\right)$ during rainy season in Ede water works system and distributed water sample $\left(29.19 \pm 0.89{ }^{\circ} \mathrm{C}\right)$ from Opa water works system during dry season. $\mathrm{pH}$ mean concentration was higher in raw water sample from both Ede and Opa water work systems $\left(7.07 \pm 0.29{ }^{\circ} \mathrm{C}, 6.67 \pm 0.32{ }^{\circ} \mathrm{C}\right)$ during the dry season. Highest nitrate mean value was recorded from treated water $(1.69 \pm 1.22 \mathrm{mg} / \mathrm{L})$ at Ede during rainy season and raw water had the highest 
nitrate mean value of $2.46 \pm 0.92 \mathrm{mg} / \mathrm{L}$ during rainy season from Opa water work system. Highest mean of phosphate $(0.02 \pm 0.04 \mathrm{mg} / \mathrm{L})$ and DO concentrations $(7.49 \pm 1.53 \mathrm{mg} / \mathrm{L}$ and $8.00 \pm 1.88$ $\mathrm{mg} / \mathrm{L}$ )were recorded from distribution water sample from both Ede and Opa water works system. The highest total heterotrophic fungal counts was recorded from treated water $(418200844 \pm 8851301134 \mathrm{cfu} / \mathrm{ml})$ from Ede water works system during dry season but was higher in distribution water sample $(22112 \pm 2029457 \mathrm{cfu} / \mathrm{ml})$ from Opa during rainy season (Table 4). The highest monthly mean of water temperature $\left({ }^{\circ} \mathrm{C}\right)$ was recorded from raw water in April both Ede and Opa waterworks system while highest $\mathrm{pH}$ value was observed in April for Ede and February for Opa waterworks. High DO mean concentration occurred in February in Ede and August for Opa while nitrate mean value was recorded in June both waterworks system. Water temperature recorded from treated water was higher in April for both Ede and Opa waterworks system while $\mathrm{pH}$ was higher in April for Ede and in February for Opa and nitrate concentration was higher in August for Ede and in June for Opa. The highest water temperature mean recorded for distribution water was higher in February for both Ede and Opa while DO value was high in June for Ede and August for Opa. Highest $\mathrm{pH}$ was observed in April for Ede and February for Opa while nitrate was recorded in August for Ede and June for Opa waterworks system (Figure 1 and 2). The highest total heterotrophic fungal counts were observed from December followed by February and least was recorded in August from Opa waterworks system (figure 3). There are two major clustered diagrams formed in Opa waterworks system showed the relationship between physico-chemical parameter and microbial quality includes DO, water temperature and showed strong relationship while nitrate, phosphate and THF correlated with each other (figure 4). Two clustered diagrams was formed from Ede waterworks system include 
THF, water temperature and $\mathrm{pH}$ showed strong correlated between them while nitrate, $\mathrm{DO}$ and phosphate showed relationship among them (figure 5). 
Table 1: Percentage occurrence of fungi species isolate from Ede-Erinle and Opa water works system

\begin{tabular}{|c|c|c|c|c|c|c|c|c|}
\hline & & Ede & & $\%$ occurrence & & Opa & & $\%$ occurrence \\
\hline & Raw & Treated & Distribution & & Raw & Treated & Distribution & \\
\hline Absidia $s p$ & & & & 0 & & + & + & 66.6 \\
\hline Aspergillus flauvs & +++ & & + & 66.6 & ++ & + & & 66.6 \\
\hline Aspergillus fumigatus & ++ & ++ & + & 100 & + & ++ & + & 100 \\
\hline Aspergillus glacaus & + & & + & 66.6 & & & & 0 \\
\hline Aspergillus niger & & & & 0 & + & & & 33.3 \\
\hline Aureobasidium oryzae & & & & 0 & & & + & 33.3 \\
\hline Aureobasidium pullularis & + & +++ & + & 100 & + & & + & 66.6 \\
\hline Cephalosporium sp. & & & + & 33.3 & & & & 0 \\
\hline Cladosporium aerbarum & & ++ & & 33.3 & & & & 0 \\
\hline Cladosporium herbarum & + & ++ & + & 100 & + & + & & 66.6 \\
\hline Cladosporium werneckii & & + & + & 66.6 & & + & & 33.3 \\
\hline Entomophthorales sp & & & & 0 & & & + & 33.3 \\
\hline Mucor aeruginosa & & + & & 33.3 & & & & 0 \\
\hline Mucor eretus & + & & & 33.3 & & & & 0 \\
\hline Mucor hiemalis & & + & + & 66.6 & & & & 0 \\
\hline Muсог тисеdo & + & & & 33.3 & & & + & 33.3 \\
\hline Mucor rammananius & + & + & & 66.6 & & & & 0 \\
\hline Neurospora silophila & & & & 0 & + & & & 33.3 \\
\hline Penicillium cannemberti & & ++ & & 33.3 & & & & 0 \\
\hline Penicillium expansium & & + & & 33.3 & & & & 0 \\
\hline Penicillium italicum & ++ & +++ & & 66.6 & & & & 0 \\
\hline Penicillium riaticum & + & & & 33.3 & & & & 0 \\
\hline Penicillium roquetfortii & + & + & & 66.6 & & & & 0 \\
\hline Rhizopus japonicas & ++ & +++++ & ++ & 100 & + & +++ & ++ & 100 \\
\hline Rhizopus oryzae & & & & 0 & ++ & ++ & + & 100 \\
\hline Rhizopus stolonifera & + & + & & 66.6 & & & & 0 \\
\hline Rhodototula sp & & + & & 33.3 & & & & 0 \\
\hline Trichoderma sp & +++ & ++ & + & 100 & ++ & ++ & + & 100 \\
\hline Trichospora brendii & + & & & 33.3 & & & & 0 \\
\hline Trichosporon brendii & & + & & 33.3 & & & & 0 \\
\hline Total & 15 & 17 & 10 & & 09 & 08 & 09 & \\
\hline
\end{tabular}


Table 2: Percentage contribution of fungal classes and species isolated from Ede-Erinle and Opa waterworks system

\begin{tabular}{|c|c|c|c|c|c|c|c|c|c|}
\hline \multicolumn{5}{|c|}{ Ede waterworks system } & \multicolumn{5}{|c|}{ Opa waterworks system } \\
\hline Class & Genus & Counts & $\begin{array}{l}\% \text { Genus } \\
\text { contribute }\end{array}$ & $\begin{array}{l}\% \text { class } \\
\text { contribution }\end{array}$ & Class & Genus & Counts & $\begin{array}{l}\% \text { Genus } \\
\text { contribute }\end{array}$ & $\begin{array}{l}\% \text { class } \\
\text { contribution }\end{array}$ \\
\hline \multirow[t]{3}{*}{ Ascomycetes } & Aspergillus & 4 & 16.67 & \multirow{5}{*}{41.6} & \multirow[t]{2}{*}{ Ascomycetes } & Aspergillus & 2 & 15.38 & \multirow[b]{3}{*}{ 23.08 } \\
\hline & Penicillium & 5 & 20.83 & & & Neurospora & 1 & 7.69 & \\
\hline & Cephalosporium & 1 & 4.17 & & \multicolumn{2}{|l|}{ Total } & 3 & & \\
\hline \multicolumn{2}{|l|}{ Total } & 10 & & & Zoopagomycetes & Entomophthorate & 1 & 7.69 & \multirow[b]{2}{*}{7.63} \\
\hline Basidiomycetes & Rhodotorula & 1 & 4.17 & & \multicolumn{2}{|l|}{ Total } & 1 & & \\
\hline \multicolumn{2}{|l|}{ Total } & 1 & & 4.17 & Eusacomycetes & Auerobasidium & 2 & 15.38 & \multirow[b]{2}{*}{15.38} \\
\hline Eusacomycetes & Auerobasidium & 1 & 4.17 & 4.17 & \multicolumn{2}{|l|}{ Total } & 2 & & \\
\hline \multicolumn{2}{|l|}{ Total } & 1 & & & \multirow[t]{3}{*}{ Zygomycetes } & Rhizopus & 2 & 15.38 & \multirow{5}{*}{30.77} \\
\hline \multirow[t]{2}{*}{ Zygomycetes } & Rhizopus & 3 & 12.5 & \multirow[b]{3}{*}{33.3} & & Mucor & 1 & 7.69 & \\
\hline & Mucor & 5 & 20.83 & & & Absidia & 1 & 7.69 & \\
\hline \multicolumn{2}{|l|}{ Total } & 8 & & & \multicolumn{2}{|l|}{ Total } & 4 & & \\
\hline Deuteromycetes & Trichoderma & 1 & 4.17 & 4.17 & Deuteromycetes & Trichoderma & 1 & 7.69 & \\
\hline \multirow[t]{3}{*}{ Dothideomycetes } & Cladosporium & 3 & 12.5 & 12.5 & \multicolumn{2}{|l|}{ Total } & 1 & & \multirow{3}{*}{$\begin{array}{r}7.63 \\
15.38\end{array}$} \\
\hline & & 4 & & & Dothideomycetes & Cladosporium & 2 & 15.38 & \\
\hline & & & & & \multicolumn{2}{|l|}{ Total } & 2 & & \\
\hline \multicolumn{2}{|l|}{ Grand total } & 24 & 100 & 100 & \multicolumn{2}{|l|}{ Grand total } & 13 & 100 & 100 \\
\hline
\end{tabular}


Table 3:Spatial physico-chemical parameter amd microbial quality of Ede-Erinle and Opa waterworks system

\begin{tabular}{|c|c|c|c|c|c|c|c|c|c|c|c|c|c|c|c|c|}
\hline & \multicolumn{8}{|c|}{ Ede Reservoir } & \multicolumn{8}{|c|}{ Opa Reservoir } \\
\hline & \multicolumn{2}{|l|}{ Raw } & \multicolumn{2}{|l|}{ Treated } & \multicolumn{2}{|l|}{ Distribution } & \multicolumn{2}{|c|}{ Anova } & \multicolumn{2}{|l|}{ Raw } & \multicolumn{2}{|l|}{ Treated } & \multicolumn{2}{|l|}{ Distribution } & \multicolumn{2}{|c|}{ Anova } \\
\hline & Mean \pm SD & Min.-Max. & Mean \pm SD & $\begin{array}{l}\text { Min.- } \\
\text { Max. }\end{array}$ & Mean \pm SD & $\begin{array}{l}\text { Min.- } \\
\text { Max. }\end{array}$ & F & $\mathrm{P}$ & Mean \pm SD & $\begin{array}{l}\text { Min.- } \\
\text { Max. }\end{array}$ & Mean \pm SD & Min.-Max. & Mean \pm SD & $\begin{array}{l}\text { Min.- } \\
\text { Max. }\end{array}$ & F & $\mathrm{P}$ \\
\hline $\begin{array}{l}\text { Water } \\
\text { temperature }\end{array}$ & $27.92 \pm 1.39$ & $26-29.50$ & $28.08 \pm 1.33$ & $\begin{array}{l}26.5- \\
30.50\end{array}$ & $28.08 \pm 0.91$ & $\begin{array}{l}26.5- \\
29.50\end{array}$ & 0.049 & 0.952 & $26.66 \pm 0.96$ & $25.5-28.0$ & $27.19 \pm 1.48$ & $25.5-29.50$ & $27.99 \pm 1.56$ & $\begin{array}{l}24.5- \\
31.00\end{array}$ & 2.091 & 0.140 \\
\hline$\overline{\mathrm{pH}}$ & $7.02 \pm 0.5$ & $6.7-7.50$ & $6.09 \pm 0.77$ & $3.8-7.40$ & $6.41 \pm 0.32$ & $5.5-7.00$ & 6.191 & 0.004 & $6.6 \pm 0.34$ & $6.1-6.90$ & $6.53 \pm 0.39$ & $6.0-7.20$ & $6.64 \pm 0.26$ & $6.2-6.90$ & 0.388 & 0.682 \\
\hline Nitrate & $0.87 \pm 0.50$ & $0.28-1.63$ & $1.03 \pm 1.09$ & $0.23-4.31$ & $0.74 \pm 0.34$ & $0.41-1.55$ & 0.635 & 0.534 & $1.71 \pm 1.22$ & $0.43-3.37$ & $1.47 \pm 1.12$ & $0.23-3.12$ & $1.31 \pm 0.99$ & $0.31-2.89$ & 0.281 & 0.757 \\
\hline Phosphate & $0.01 \pm 0.01$ & $0.002-0.02$ & $0.01 \pm 0.004$ & $\begin{array}{l}0.001- \\
0.02\end{array}$ & $0.018 \pm 0.03$ & $\begin{array}{l}0.002- \\
0.10\end{array}$ & 2.018 & 0.145 & $0.01 \pm 0.01$ & $0.00-0.02$ & $0.01 \pm 0.01$ & $0.01-0.02$ & $0.012 \pm 0.01$ & $\begin{array}{l}0.002- \\
0.03\end{array}$ & 0.276 & 0.761 \\
\hline DO & $7.13 \pm 1.14$ & $5.2-8.40$ & $6.9 \pm 1.02$ & $4.8-8.80$ & $7.08 \pm 1.93$ & $3.6-11.20$ & 0.109 & 0.897 & $4.0 \pm 1.23$ & $3.2-6.0$ & $6.77 \pm 1.83$ & $4.4-10.40$ & $8.1 \pm 1.99$ & $4.4-12.40$ & 9.784 & 0.000 \\
\hline THF & $\begin{array}{l}12290 \pm \\
26671.95\end{array}$ & $150-60000$ & $246 \pm 215.59$ & $20-600$ & $8575 \pm 20294$ & $\begin{array}{l}100- \\
50000\end{array}$ & 0.507 & 0.614 & $\begin{array}{l}422550 \pm \\
1017803.59\end{array}$ & $\begin{array}{l}500- \\
2500000\end{array}$ & $\begin{array}{l}209108461 \pm \\
629904535.78\end{array}$ & $\begin{array}{l}160- \\
2000000000\end{array}$ & $\begin{array}{l}22670205.56 \pm \\
34089142.19\end{array}$ & $\begin{array}{l}50- \\
73000000\end{array}$ & 0.709 & 0.503 \\
\hline
\end{tabular}

Table 4: Seasonal physico-chemical parameter amd microbial quality of Ede-Erinle and Opa waterworks system

\begin{tabular}{|c|c|c|c|c|c|c|c|c|c|c|c|c|}
\hline \multirow[t]{3}{*}{ Parameters } & \multicolumn{4}{|c|}{ Raw } & \multicolumn{4}{|c|}{ Treated } & \multicolumn{4}{|c|}{ Distribution } \\
\hline & \multicolumn{2}{|c|}{ Dry } & \multicolumn{2}{|c|}{ Rainy } & \multicolumn{2}{|c|}{ Dry } & \multicolumn{2}{|c|}{ Rainy } & \multicolumn{2}{|c|}{ Dry } & \multicolumn{2}{|c|}{ Rainy } \\
\hline & Ede & Opa & Ede & Opa & Ede & Opa & Ede & Opa & Ede & Opa & Ede & Opa \\
\hline $\begin{array}{c}\text { Water } \\
\text { temperature }\end{array}$ & $29.00 \pm 0.86$ & $28.00 \pm 1.01$ & $26.83 \pm 0.76$ & $26.10 \pm 0.66$ & $29.21 \pm 0.78$ & $28.3 \pm 1.29$ & $26.95 \pm 0.56$ & $26.08 \pm 0.42$ & $28.56 \pm 0.95$ & $29.19 \pm 0.89$ & $27.61 \pm 0.60$ & $26.80 \pm 1.08$ \\
\hline $\mathrm{pH}$ & $7.07 \pm 0.29$ & $6.67 \pm 0.32$ & $6.97 \pm 0.46$ & $6.43 \pm 0.35$ & $6.11 \pm 0.81$ & $6.45 \pm 0.44$ & $6.07 \pm 0.76$ & $6.62 \pm 0.35$ & $6.48 \pm 0.29$ & $6.51 \pm 0.29$ & $6.36 \pm 0.35$ & $6.54 \pm 0.34$ \\
\hline Nitrate & $0.46 \pm 0.18$ & $0.60 \pm 0.15$ & $1.27 \pm 0.33$ & $2.46 \pm 0.92$ & $0.37 \pm 0.11$ & $1.07 \pm 1.03$ & $1.69 \pm 1.22$ & $1.88 \pm 1.15$ & $0.49 \pm 0.10$ & $0.98 \pm 0.94$ & $0.99 \pm 0.32$ & $1.64 \pm 0.99$ \\
\hline Phosphate & $0.01 \pm 0.01$ & $0.01 \pm 0.01$ & $0.01 \pm 0.01$ & $0.01 \pm 0.01$ & $0.01 \pm 0.01$ & $0.01 \pm 0.01$ & $0.01 \pm 0.01$ & $0.01 \pm 0.01$ & $0.02 \pm 0.04$ & $0.01 \pm 0.01$ & $0.01 \pm 0.01$ & $0.01 \pm 0.01$ \\
\hline DO & $7.47 \pm 1.01$ & $4.00 \pm 0.69$ & $6.80 \pm 1.39$ & $4.13 \pm 1.62$ & $6.97 \pm 0.86$ & $7.27 \pm 2.28$ & $6.83 \pm 1.99$ & $6.27 \pm 1.26$ & $6.67 \pm 2.27$ & $8.20 \pm 2.20$ & $7.49 \pm 1.53$ & $8.00 \pm 1.88$ \\
\hline THF & $\begin{array}{l}834700 \pm \\
1442192.56\end{array}$ & $\begin{array}{l}12290.0 \pm \\
26671.95\end{array}$ & $\begin{array}{l}10400 \pm \\
16974.39\end{array}$ & $\begin{array}{l}30100 \pm \\
42284.99\end{array}$ & $\begin{array}{l}418200844 \pm \\
885130113.4\end{array}$ & $\begin{array}{l}246.00 \pm \\
215.59\end{array}$ & $\begin{array}{l}16078.00 \pm \\
16640.07\end{array}$ & $180.0 \pm 28.28$ & $\begin{array}{l}51000100 \pm \\
34244022\end{array}$ & $8575 \pm 20294.57$ & $6290 \pm 13255.58$ & $22112 \pm 20294.57$ \\
\hline
\end{tabular}


Table 5: Correlation matrix showing the relationship between THF and water quality

\begin{tabular}{|l|l|l|l|l|l|l|}
\hline & \multicolumn{5}{l}{ Ede-Erinle water works system } & \multicolumn{3}{l|}{ Opa water works system } \\
\hline Water quality & Raw & Treated & Distribution & Raw & Treated & Distribution \\
\hline Water temperature & $0.56^{*}$ & -0.26 & 0.20 & 0.30 & $-0.58^{* *}$ & -0.01 \\
\hline pH & -0.16 & 0.12 & -0.13 & 0.29 & -0.07 & -0.32 \\
\hline Nitrate & $-0.57^{* *}$ & 0.17 & -0.32 & -0.35 & 0.40 & -0.04 \\
\hline phosphate & $-0.54^{*}$ & 0.02 & $0.69^{* *}$ & 0.13 & -0.21 & 0.27 \\
\hline DO & $0.53^{*}$ & 0.41 & 0.14 & -0.04 & -0.32 & $-0.47^{*}$ \\
\hline
\end{tabular}




\section{Ede Raw water}

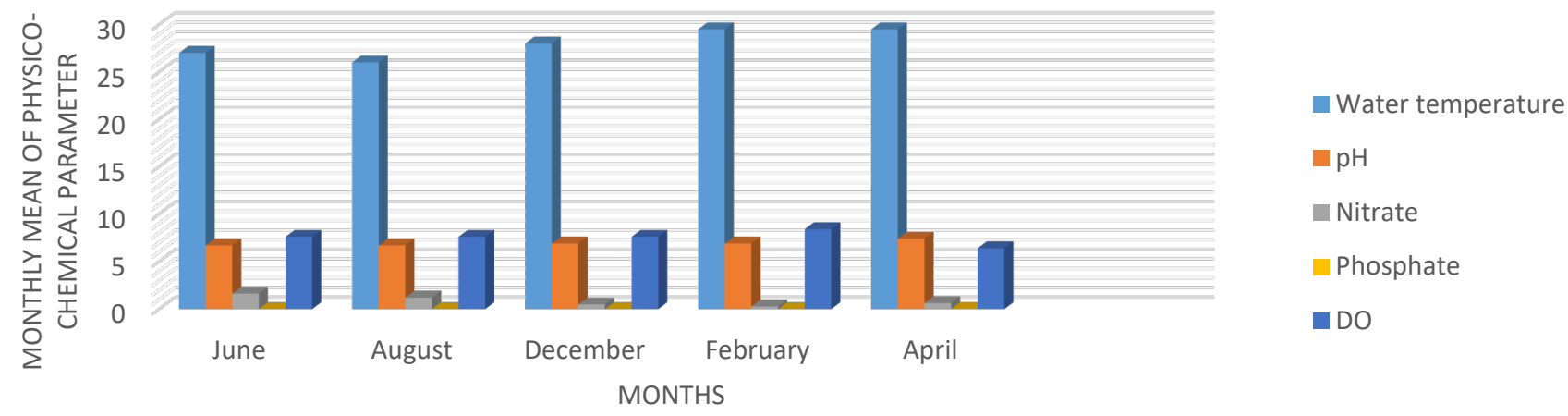

\section{Opa Raw water}
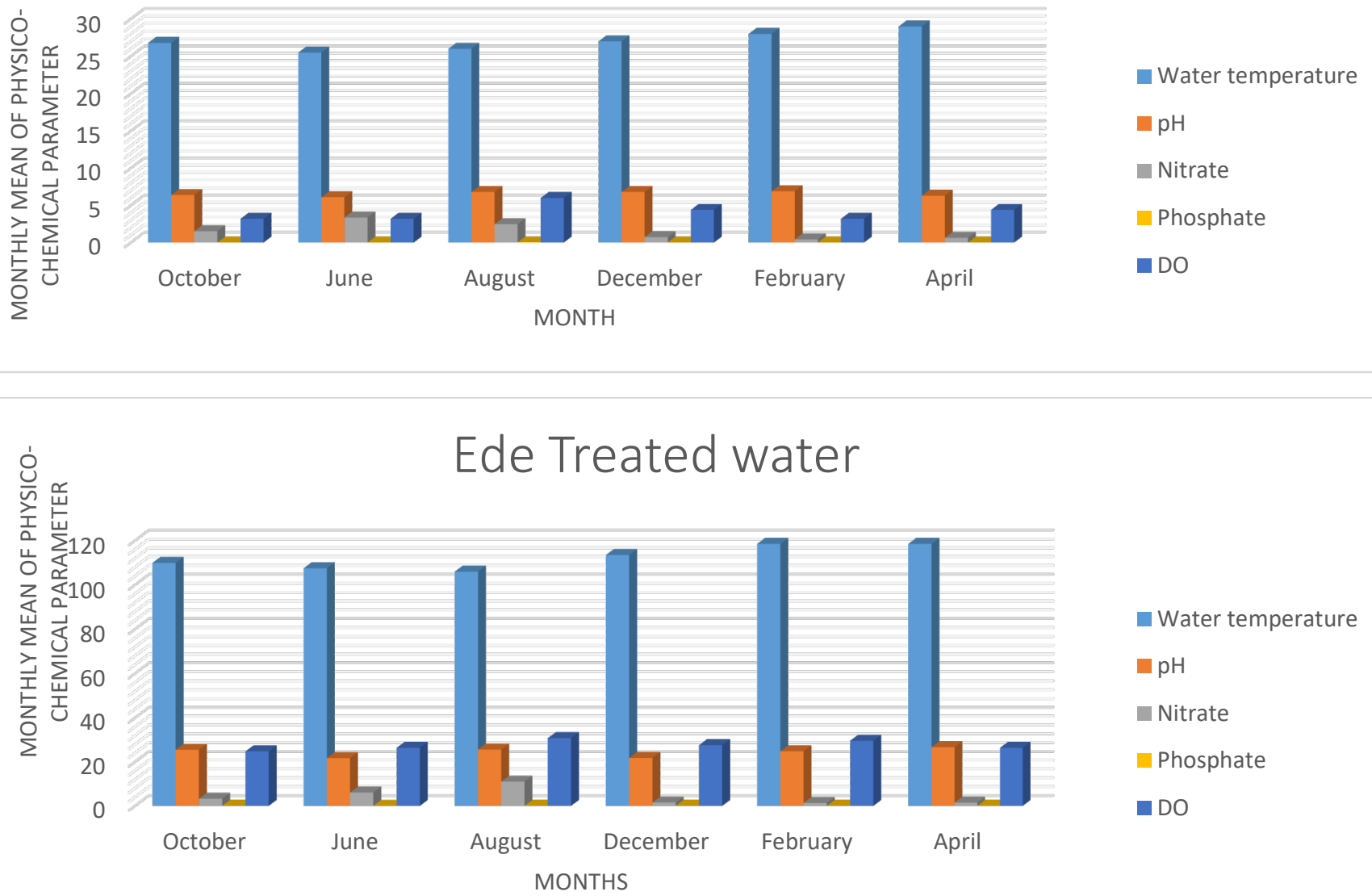

Figure 2: 


\section{Opa Treated water}

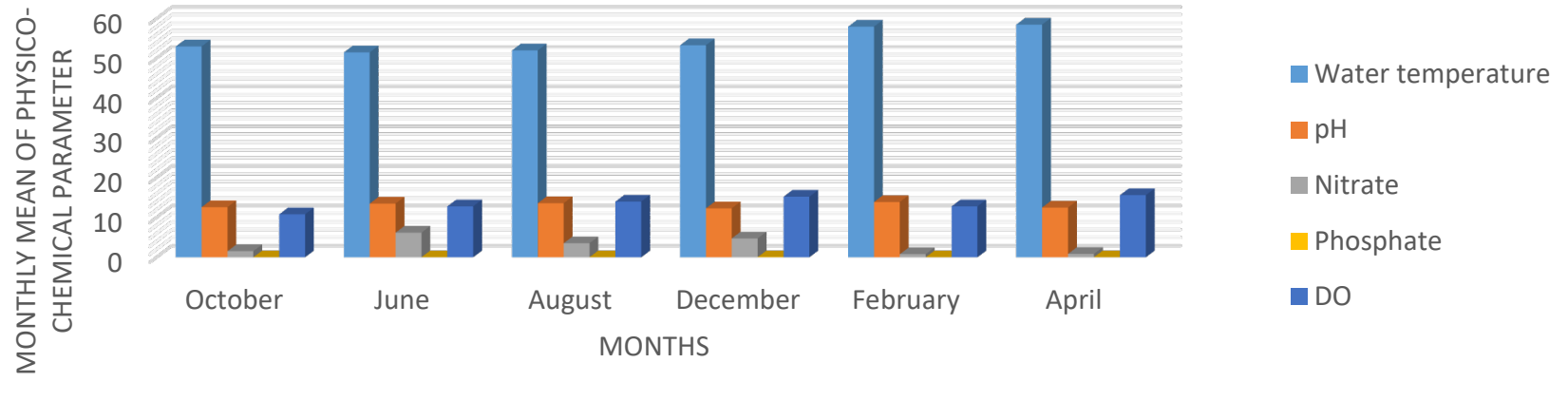

\section{Ede Distribution water}

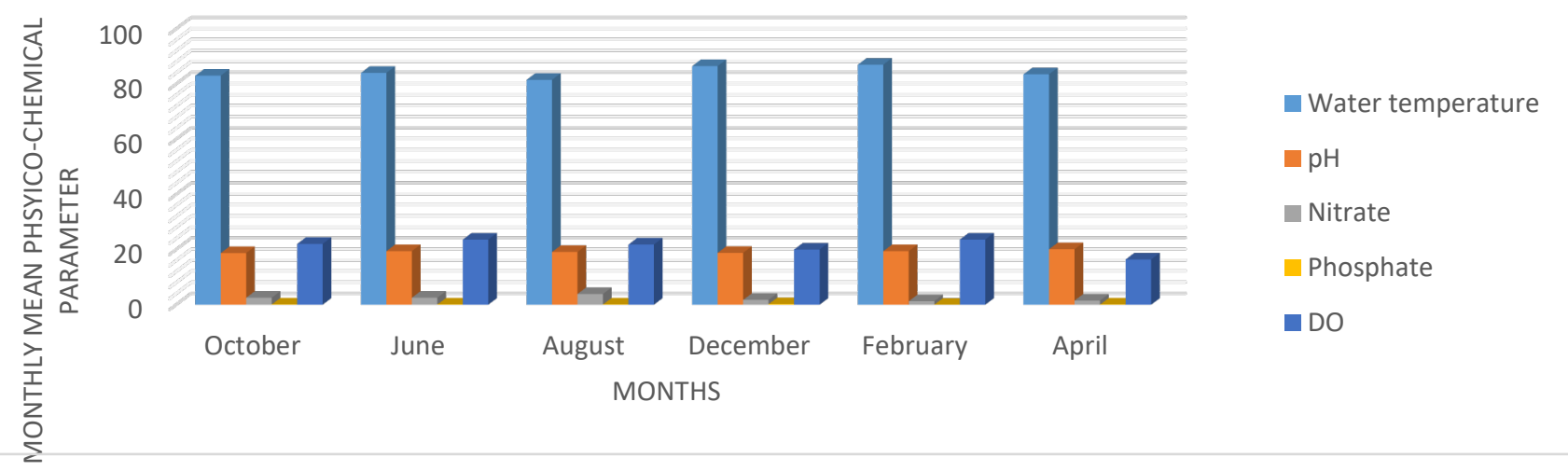

\section{Opa Distribution water}

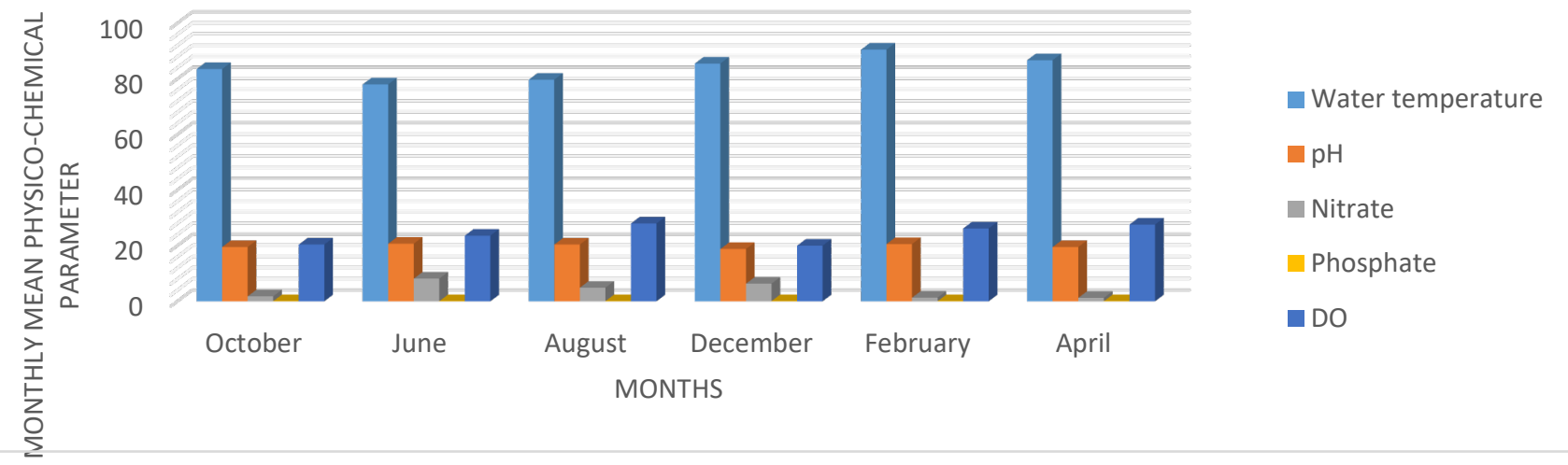

Figure 2 


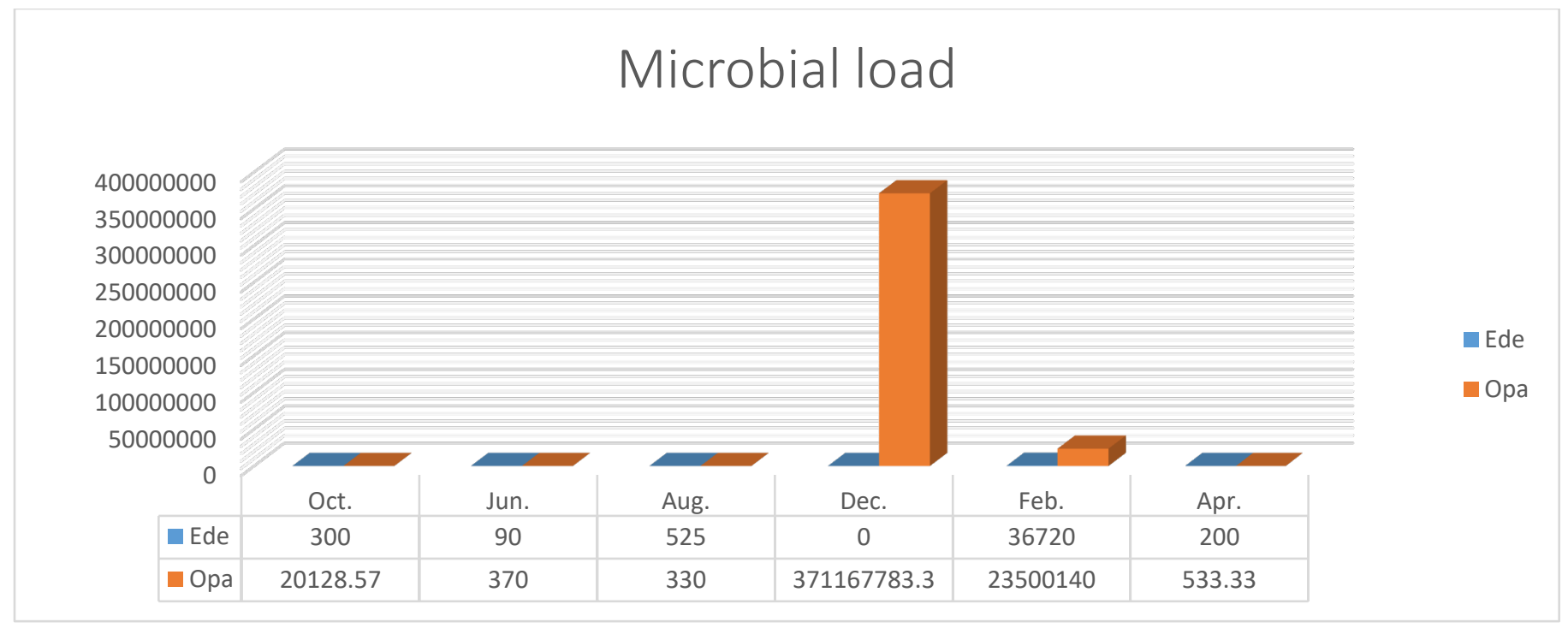

Figure 3: Abundances of Microbial quality in Ede-Erinle and Opa Waterworks system 


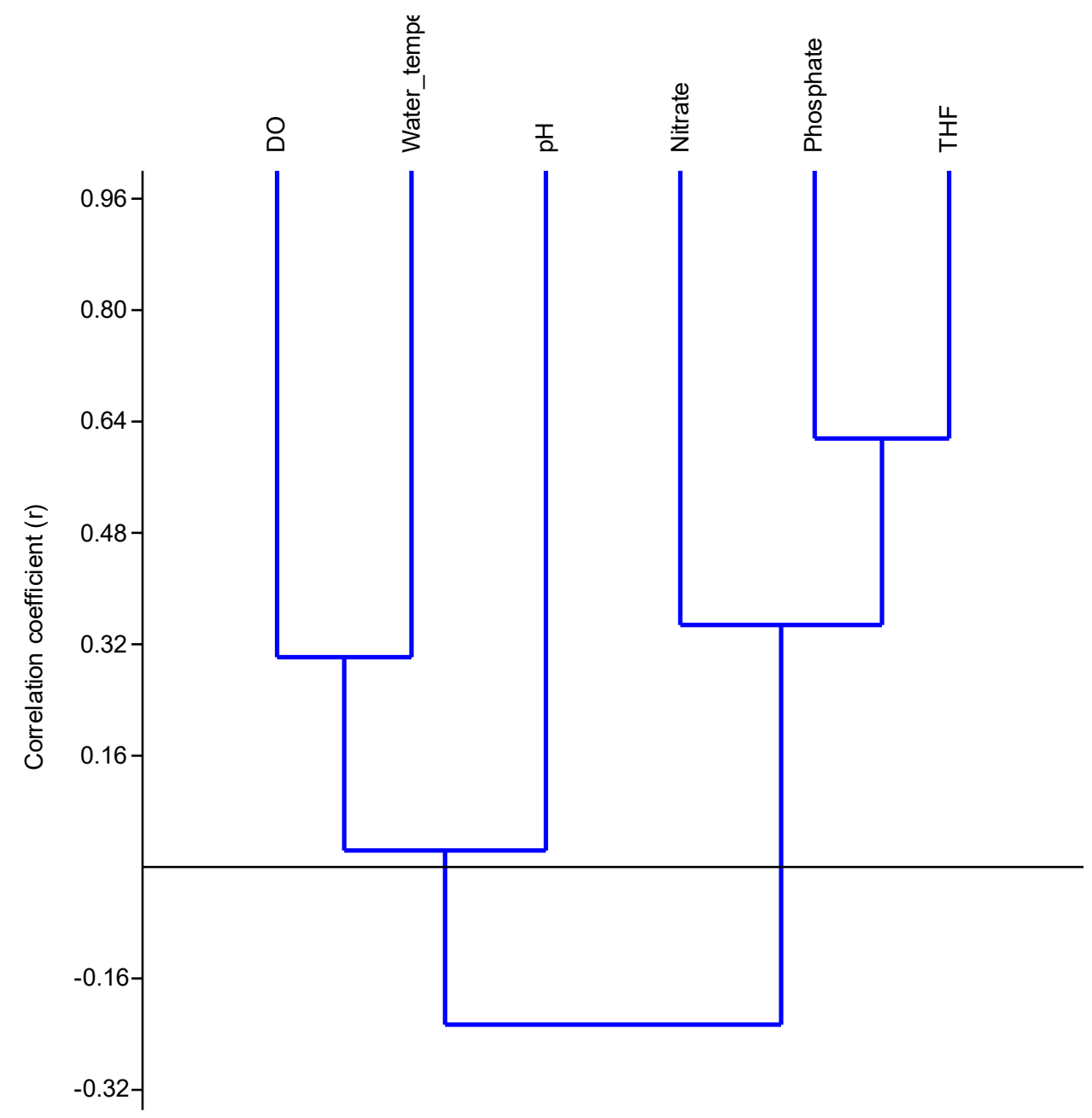

Figure 4: Cluster diagram showing relationship between physico-chemical parameter and microbial quality of Opa waterworks system 


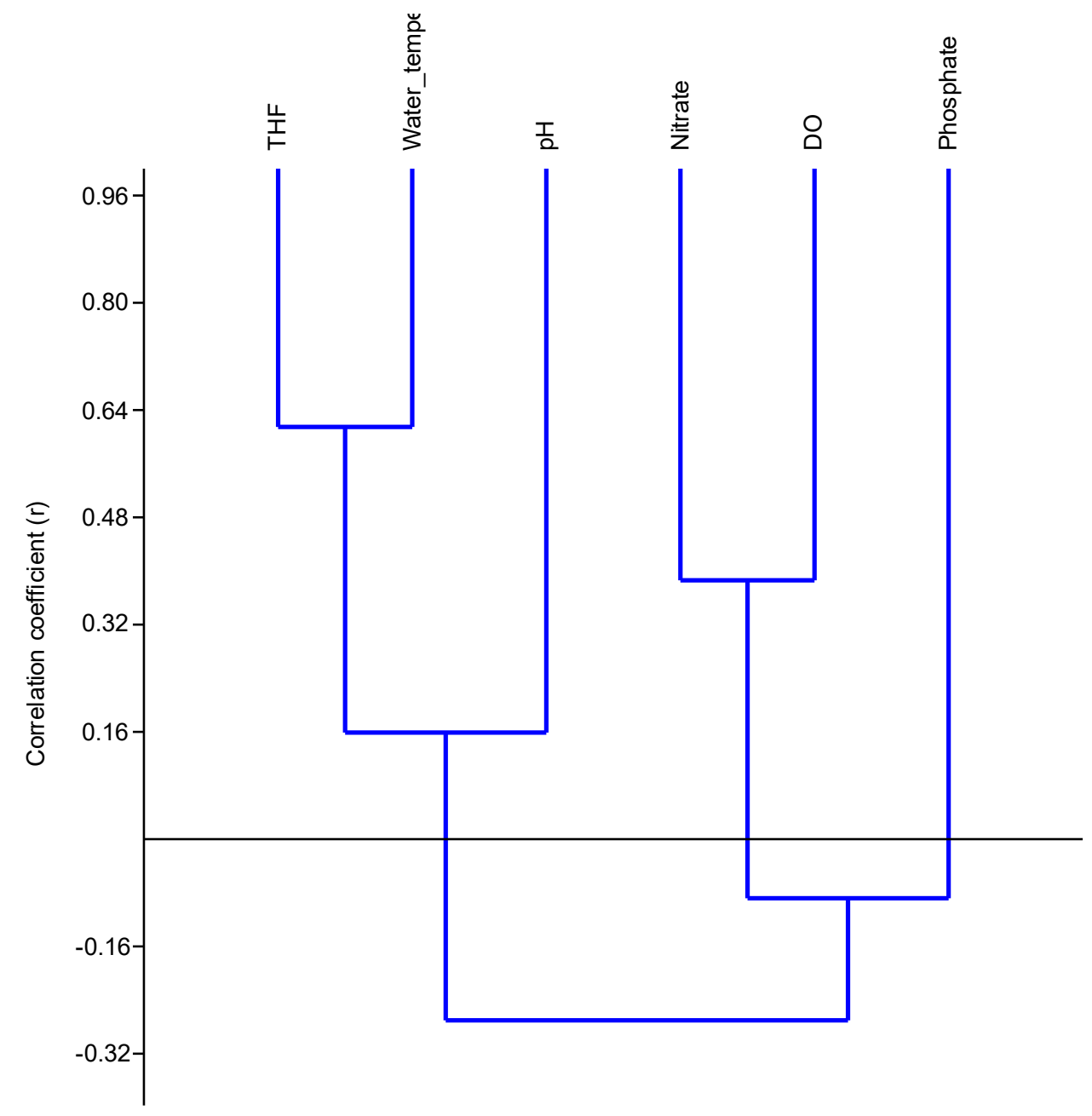

Figure 5: Cluster diagram showing relationship between physico-chemical parameter and microbial quality of Ede-Erinle waterworks system 


\section{Discussion}

Ascomycete are most dominant family observed from Ede-Erinle water works system followed by zygomycetes and dothideomycete while Opa water works system were dominant by Zygomycete follow by Ascomycetes and Eusacomycetes. Many people who use this water are prone to waterborne diseases because ascomycetes are pathogens of humans, animals and plants such as they cause infections in humans include Candida albicans, Aspergillus niger and several tens of species that cause skin infections. There are two orders of Zygomycetes containing organisms that cause human disease, the Mucorales and the Entomophthorales. The majority of human illness is caused by the Mucorales while most diseases are most commonly linked to Rhizopus spp., other organisms are also associated with human infection, including Mucor, Rhizopus, and Absidia that were recorded in this study. Human zygomycosis caused by the Mucorales generally occurs in immunocompromised hosts as opportunistic infections. Host risk factors include diabetes mellitus, neutropenia, sustained immunosuppressive therapy, chronic prednisone use, iron chelation therapy, broad-spectrum antibiotic use, severe malnutrition, Cutaneous, gastrointestinal, and allergic diseases. The Mucorales are associated with angioinvasive disease, often leading to thrombosis, infarction of involved tissues, and tissue destruction mediated by a number of fungal proteases, lipases, and mycotoxins. Not all fungi produce mycotoxins, as most mycotoxin producing species are filamentous ascomycetes, basidiomycetes and Deuteromycetes with Penicillium, Aspergillus and Fusarium being the most mycotoxin-producing genera (Abbott 2002).

The high fungi occurrence observed from both raw water in Ede-Erinle and Opa reservoirs might be due to high influx of runoffs that contain high fungi load from tributaries river that flow into those reservoir. Fungi are natural inhabitants of composting plants, soil and water but they are 
capable of migrating from terrestrial environment and end up in fresh water systems through runoff (Wurzbacher et al. 1998; Magwaza et al. 2017). The influence of anthropogenic activities, abiotic and biotic factors that favor microbial growth in water sources such as location of the water source, water flow, ion composition, presence of organic matter, pollution rate, and water cleaningprocesses can also contribute to high fungal species isolated recorded from this study (Novak et al. 2017). Fungi genera like Aspergillus, Aureobasidium, Cladsporium, Mucor, Penicillium, Rhizopus, Trichoderma, Trichospora and Neurospora have the highest occurrences in the raw water that can caused waterborne diseases for those drinking raw water whether treating it. Kanzleret al. 2007 reported that fungal species like Fusarium and Aspergillus species have been identified to multiply in water reservoirs and causes waterborne infections. The consumption of drinking water polluted by fungi had been associated with serious disease casualties (Novak et al. 2017).

The total heterotrophic fungi mean observed in Opa treated water was higher than Ede-Erinle treated water might be duetochemical-physical characteristics like high turbidity and temperature, $\mathrm{pH}$, total organic carbon and dissolved oxygen, are favourable to microbial growth, making these environments at potential risk of water quality degradation by fungi (Oliveira et al. 2016). Based on report of Sammon et al. (2011) and Pereira et al. ( 2013) has revealed c, including fungi in water that end up in treated water distribution systems to the consumer point of use. The presence of fungi species such as Absidia sp., Aureobasidium sp., Cladosporium sp.,Mucor sp., Rhizopus sp., Rhodototula sp., Penicillium sp., Aspergillus sp., Trichoderma sp., and Trichosporon sp. from Ede-Erinle and Opa water works treatment plant, are capable of producing mycotoxins and other secondary metabolites that produce toxic chemicals which impair water quality and become a threat to humans and animals (Sonigo et al. 2011). 
Aspergillus spp vary with regards to toxin and metabolite production, temperature of growth, and other parameters that dictate pathogenicity and can cause illness for consumer of this water due to Aspergillosis infection usually affects the respiratory system but their sign severity vary greatly cause serious illness when people with weakened immune system, underlying lung disease or asthma or/ cystic fibrosis, low white blood cell level, long-term corticosteroid therapy. They can survive during water treatment because these organism secrets multiple toxins and metabolites that protect it forms and it has specific cell wall components that can serve to promote survival in the host as well. Cladosporium are not dangerous to human but long-term exposure to a large amount of any mold including Cladosporium can adverse health effects on humans such allergies and asthma symptom and other aliments including eye, ear, skin and sinus infections. Severe shortness of breath, difficulty breathing, dry skin, hives, sneezing, watery eyes, postnasal drip, long-term nasal congestion, recurring sinus headaches, chest tightness and pain. Mucor contaminated water or food constitutes a limited potential health hazard with regard to healthy consumers. Despite this toxin production, the genus mucor is generally accepted to be non-toxic toward humans. Mucor fungi cause the group of infection referred to as zygomycoiss or mucormycosis includes mucocutaneous and rhinocerebral infection as well as septic arthritis, dialysis-associated peritonitis, renal infection, gastritis and pulmonary infection. Penicillium spp are known to produce mycotoxin. They are occasional causes of infection in human and the resulting disease is known generically as penicilliosis.

Most of the fungal genera described in the studies are dematiaceous fungi that are capable of secreting melanin or melanin-like pigment in their cell walls. This makes them thick-walled species with hydrophobic spores that give them the advantage to resist water treatment (Auwal and Taura 2013; Al-gabr et al. 2014; Babič et al. 2017). Treated drinking water quality without 
pathogenic microorganisms including fungi is very critical for the health of all humans and mostly those with immunodeficiency conditions. An increasein population of patients having an impaired immune system, as their immune effector cells become compromised allowing fungi to colonize and attack the human tissues, leading to more complications (Oliveira et al. 2013). Fungi cause number of diseases including allergies, respiratory illness, cutaneous infection and life-threatening meningitis (Sulaiman et al. 2014). Mycotoxigenic fungi have been reported in treated drinking water (Paterson and Lima 2008; Al-gabr et al. 2014). Many methods using in the water treatment are note effective for fungi removal or killing them during the process (Hageskal et al. 2009). Fungi and other bacteria have the ability to become dormant in order to preserve their lives when conditions are no longer conducive. Nevertheless, become active when condition is favourable and return to their normal state, recovering their metabolic activity and starting the process of spore germination (Luu et al. 2015). Fungi can survive sedimentation and flocculation, rapid sand filtration does not become an effective treatment for fungi (Kinsey et al. 2003) as filters shown partially remove microorganisms and end up in the distribution system (Kinsey et al. 2003). The most trusted treatment process for destroying pathogenic microorganisms is disinfection but most microorganisms have developed resistance against it (Tellen et al. 2010). The use of disinfection in water treatment as a public health measure has shown a major decline in people contracting water-related diseases from drinking water (EPA 2013). Penicillium and Aspergillus species are more resistant to chlorine disinfection than the Cladosporium and Phoma species (Pereira et al. 2013). Ozone and UV radiation are more capable in the destruction of many pathogenic organisms than chlorine, but their disadvantages are high costs and mostly the inability to have residual that persists long enough to prevent the re-growth. There are resistant species to ozone like Trichoderma viride that is slightly affected 
only in elevated concentrations and Penicillium spinulosum which is the most resistant due to its hydrophobic surface (Hageskal et al. 2012).Fungi with pigmented spores such as Aspergillus and Penicillium have better defense against radiation and are not responsive to UV treatment (Hageskal et al. 2009). The radiation cannot destroy fungal species even in slightly turbid water, as the fungi tend to be harboured within the particles and escape disinfection (EPA 2013). Pathogenic fungi are believed to have caused hostile infections that have contributed to high mortality rates (Arvanitidou et al. 1999; Khan et al. 2010; Mayer et al. 2013; Tsui et al. 2016; Pal 2017). Fungal deposition in water distribution systems is attributed to spores and not hyphae growth, which raises concerns that mycotoxins' production of taste and odour problems suggest that vegetative growth, occurs in situ (Doggett 2000). Microorganisms enter the distribution system after surviving water treatment processes, and due to recontamination and re-growth (Liu et al. 2016). There is high abundance of total heterotrophic fungi counts in distribution station from Opa water works system was greatly higher than distribution station in Ede water works. The presence of genera such as Aspergillus, Aureobasidium, Cephalosporium, Mucor, Rhizopus, Trichoderma, Entomophthorales were encounter from the distribution stations in both Ede and Opa water works system are mycotoxins fungal which produced poisons as secondary metabolites by the mycelial structure of filamentous fungi as well as spores (Gupta et al. 2015). Cladosporium sp., Aspergillus sp., Penicillium sp. and Fusarium sp. have been linked to allergies and respiratory illness (Máizet al. 2018). Taste and odour problems in water are caused by Aspergillus sp., Acremonium sp., Phialophora sp. and Penicillium sp. (Sonigoet al. 2011). Fungi such as Rhizopus, Fusarium, Alternaria, Aspergillus and Penicillium produce mycotoxins that are harmful to public health as these mycotoxins are carcinogenic and have the ability to impair the immune system (Magwazaet al. 2017). Mycotoxin concentrations can be low in water 
because of dilution, water retention in storage tanks, the long distances water travels in distribution systems, depletion of chlorine residual and the resistance of some of the fungi to disinfection can cause mycotoxin concentrations to increase to unsafe amounts in drinking water (Barrigaet al. 2012). Small amounts of mycotoxins consumed regularly could be harmful to human health (Hageskalet al. 2009).The types of infections caused by mycotoxigenic fungi depend on the type of mycotoxin, the concentration and length of exposure; as well as age, health, and sex of the exposed individual (Bennett and Klich 2003). The absence of toxigenic fungi in treated drinking water may not provide assurance that the water is free of mycotoxins, as mycotoxins may persevere long after the fungi has died (Pitt et al. 2000). Mycotoxins have serious and chronic effects on humans and animals, as many of them are believed to be carcinogenic, cytotoxic, mutagenic and may lead to immunosuppressive complexes (ArroyoManzanareset al. 2015).

\section{Water quality}

Microorganisms are profoundly affected by environmental and physic-chemical factors including temperature, aeration, $\mathrm{pH}$, water potential, light and adaptation to extreme environmental conditions. These factors not only affect the growth rate of fungi but also can act as triggers in developmental pathways. The mean of water temperature recorded from Ede and Opa water works system were within WHO (2015) recommended standard of 27 to $28^{\circ} \mathrm{C}$ for drinking water expect for Opa raw water which was lower than stipulated limit might be attributed to the sampling time and climatic factor. low mean water temperature were also observed from Ede and Opa raw water samples, Opa treated and distribution water samples could be due to shading, water body size and depth, time of year and time of day which was similar to report of Atobatele and Ugwumba, (2008). There are strong correlation between water 
temperature, DO and total heterotrophic fungi counts which shown temperature have effect on the abundance of total heterotrophic fungi counts from Ede water works system. The ranged of water temperature recorded from various water samples support the growth and development of some fungi species such as Aspergillus sp., Cladosporium sp., and Pencillium sp., that grow at optimum temperature ranging from 10 to $40^{\circ} \mathrm{C}$.

The $\mathrm{pH}$ mean values recorded from both Ede and Opa water works system are lower than permissible limit of WHO (2015) and SON (2007) for portable water of 6.5-8.5 as well as for Opa and Ede seasonally mean values. The result obtained in this study could be to due to predominant soil type in the area or possibly to the built-up of organic material from runoff in raw water. As organic substances decay, carbon dioxide is released and combines with water to produce weak acid "carbonic" acid. Accumulation of free carbon dioxide due to little photosynthetic activities of phytoplankton will lower the $\mathrm{pH}$ value of the water while intense photosynthetic activities of phytoplankton will reduce the free carbon dioxide content resulting in increased $\mathrm{pH}$ values (Gupta and Gupta, 2006). The low $\mathrm{pH}$ mean concentration observed in treated water might be due to the process the water through while in the distribution could be due to corrosive of the pipes or leaking of this pipes that transporting the water to various distribution station that reaction with water to make it acidic. With ranged of $\mathrm{pH}$ concentration observed from this study could be as result of high fungi recorded. Parameters such as temperature and $\mathrm{pH}$ also encourage growth and persistence of fungi in water (Hussain et al. 2011). Many fungi will grow over the $\mathrm{pH}$ range $4.0-8.5$, or sometimes $3.0-9.0$, and they show relatively broad $\mathrm{pH}$ optima of about 5.0-7.0.

High mean concentration of dissolved oxygen (DO) from both Ede and Opa water works system could be attributed to aeration from the high stream flow rate at the time when the samples were 
collected (Ayoade et al, 2006). High DO mean values was recorded during the dry season high mean concentration of DO was observed than during the wet season expect in Opa raw water. The mean results of DO recorded during both season were within the minimum value of 5.0 milligrams per liter $(\mathrm{mg} / \mathrm{L})$ of DO standard for surface water and this shows correlations with the works of Atobatele and Ugwumba (2018). High DO values observed from this present study can also encourage growth of fungi in water because most fungi are strict aerobes, since they require oxygen in at least some stages of their life cycle such as Fusarium oxysporum, Mucor hiemalis, Aspergillus fumigatus that are facultative aerobes but also can grow in the absence of oxygen

Nitrate and phosphate containing compounds create serious problem when it high above the standard in water bodies and they are major nutrients needed by living microorganisms for their physiological processes. However, they are considered as pollutants if their concentration is more than recommended limit. The mean values of nitrate and phosphate recorded from Opa and Ede water works system were below WHO (2015) stipulated standard for drinking water. Clustered diagram showed there is close relationship between nutrient elements and total heterotrophic fungi counts might result to low abundance of fungi species recorded in Opa water works system. Nitrate contaminated water is hazardous especially for the infants and pregnant women (Chen, 1996). It may causes 'methemoglobinemia' or blue baby syndrome and it is highly harmful for infants (Shih et al., 1997). Nitrosamine is carcinogenic in nature and directly affects the esophagus and pharynx. Prolong use of nitrate contaminated drinking water causes prostrate and gastrointestinal cancer (Morales-Suarez-Varela et al., 1995). It may cause abortion due to deficiency of oxygen in the fetus in pregnant women (Grant et al., 1996). Studies have also revealed that long-term exposure to nitrate levels between 11 and 
$61 \mathrm{mg} / \mathrm{L}$ could increase the risk of hyper thyroidism (Seffener, 1995), while nitrate levels greater than $25 \mathrm{mg} / \mathrm{L}$ have been associated with insulin dependent diabetes. The recommended value of phosphorous in drinking water according to EPA is $5 \mathrm{mg} / \mathrm{l}$. Though, in permissible limit it is essential for human health, however, beyond permissible limit it may damage kidney and causes osteoporosis.

\section{Conclusion}

The presences of mycotoxigenic fungal such as Aspergillus sp, Mucor sp, Cladosporium sp., Penicillium sp., and Trichoderma sp from the treated and distribution water from Ede and Opa water work system after the water as undergo treatment process is unacceptable, and could pose serious health risks to consumers because they can causes aspergillosis, mucormycosis and penicilliosis infection in human. Prolong research needs to be conducted by the management on how effectiveness of the techniques using in water treatment at both Ede and Opa Water work's system to minimize or control the growth of these mycotoxigenic fungal in the water work's.

since some of this fungal species can survive water treatment process and more detail of the environmental factors that can aid their growth in water should also be carried-out. 


\section{REFERENCES}

Abbott, S. P. (2002). Mycotoxins and indoor molds. Indoor Environment Connections 3 (4), 1424.

Adam, K., Heath, R. G. M. and Steynberg, M. C. (2017). Invertebrates as biomonitors of sandwater efficiency. Water SA 24 (1), 43-48.

Al-gabr, H. M., Zheng, T. and Yu, X. (2014). Occurrence and quantification of fungi and detection of mycotoxigenic fungi in drinking water in Xiamen City, China. Science of the Total Environment 466-467, 1103-1111.

Arroyo-Manzanares, N., Huertas-Pérez, J. F., Gámiz-Gracia, L. and García-Campaña, A. M. (2015). Simple and efficient methodology to determine mycotoxins in cereal syrups. Food Chemistry 177, 274-279.

Atanda, S. A., Pessu, P. O., Agoda, S., Isong, I. U., Adekalu, O. A., Echendu, M. A. and Falade, T. C. (2000). Fungi and mycotoxins in stored foods. African Journal of Microbiology Research 5 (25), 4373-4382.

Auwal, H. and Taura, D. (2013). Prevalence of moulds in households drinking water of some local government areas of Kano. Nigeria. Greener Journal of Biological Sciences 3 (5), 179-186.

Ayodele, H. A. (1979). The influence of some Physico-Chemical factors on the composition, abundance and character of zooplankton in some lakes in Osun River Basin. M.Sc. Thesis. University of Ife, Nigeria.

Babic, M. N., Zalar, P., Ženko, B., Džeroski, S. and Gunde-Cimerman, N. (2017). Yeasts and yeast-like fungi in tap water and groundwater, and their transmission to household appliances. Fungal Ecology 20, 30-39.

Barnett, H. L. and Hunter, B. B. (1998). Illustrated Genera of Imperfect Fungi. APS Press, Minnesota, USA. pp 218

Barriga, L. F. S., León, A. J. M., Narvaez, D., Pérez, M. F. L., Groot, H. and Rodríguez, M. S. S. (2012). Toxicological and Products Formation Approach of Fungal Organic Matter From Drinking Water Distribution System. Environmental Engineering Research Center, Bogotá, Colombia 
Bennett, J. W. and Klich, M. (2003). Mycotoxins. Clinical Microbiology Reviews 16 (3), 497516.

Bhat, R., Rai, R. V. and Karim, A. A. (2010). Mycotoxins in food and feed: present status and future concerns. Comprehensive Reviews in Food Science and Food Safety 9 (1), 5781.

Black, K. E. and Baden, L. R. (2007). Fungal infections of the CNS: treatment strategies for the immunocompromised patient. CNS Drugs 21 (4), 293-318.

Calvo-Polanco, M., Sánchez-Castro, I., Cantos, M., García, J. L., Azcón, R., Ruiz-Lozano, J. M., and Aroca, R. (2016). Effects of different arbuscular mycorrhizal fungal backgrounds and soils on olive plants growth and water relation properties under well-watered and drought conditions. Plant, cell \& environment, 39(11), 2498-2514.

Campbell, A. W., Thrasher, J. D., Gray, M. R. and Vojdani, A. (2004). Mold and mycotoxins: effects on the neurological and immune systems in humans. Advances in Applied Microbiology 55, 375-406.

Cheesbrough, M (2000). District Laboratory Practice in Tropical Countries Part 2, Cambridge University Press, Cambridge. pp 47-54

DEFRA (Department for Environment, Food and Rural Affairs) (2011). A Review of Fungi in Drinking Water and the Implications for Human Health. 1st ed. BIO Intelligence Service; Paris, France: 2011.p. 107.

Doggett, M. S. (2000). Characterization of fungal bioflms within a municipal water distribution system. Applied and Environmental Microbiology 66 (3), 11-14.

Egborge, A. B. M. (1979). Observations on the diurnal changes in some physicochemical variables of Lake Asejire, a new impoundment in Nigeria. Pol. Arch. Hydrobiol, 26: 301311.

Egborge, A. B. M. (1981). The composition, seasonal variation and distribution of zooplankton in Lake Asejire, Nigeria. La Revue de Zoologic Africaine, Vol. 95. FASC. (1-1981). pp 137- 165

Egborge, A.B.M. (1979). The Seasonal Variation and Distribution of Phytoplankton in the River Oshun, Nigeria. Freshwater Biology, 4, 177-191.

Fox, A. R., Houser, K. H., Morris, W. R. and Walton, R. C. (2016). Dematiaceous fungal endophthalmitis: report of a case and review of the literature. Journal of Ophthalmic Inflammation and Infection $6(1), 43$.

Grabin'ska-Loniewska, A., Konillowicz-Kowalska, T., Wardzyn'ska, G. and Boryn, K. (2007). Occurrence of fungi in water distribution system. Polish Journal of Environmental Studies $16(4), 539-547$. 
Gray F.N. (2014). Pathogen control in drinking water. In: Percival L.S., Yates V.M., editors. Microbiology of Waterborne Diseases. 2nd ed. Volume 1. Elsevier; Oxford, UK: 2014. pp. $537-570$

Gupta, V. K., Mach, R. L. and Sreenivasaprasad, S. (2015). Fungal Biomolecules: Sources, Applications and Recent Developments. John Wiley and Sons, Chichester, UK, pp. 153160.

Hageskal, G., Knutsen, A. K., Gaustad, P., De Hoog, G. S. and Skaar, I. (2006). Diversity and significance of mold species in Norwegian drinking water. Applied and Environmental Microbiology 72 (12), 7586-7593.

Hageskal, G., Lima, N. and Skaar, I. (2009). The study of fungi in drinking water. Mycological Research 113 (2), 165-172.

Hageskal, G., Lima, N. and Skaar, I. (2009). The study of fungi in drinking water. Mycological Research 113 (2), 165-172. https://doi.org/10.1111/j.1365-2427.1974.tb00088.

Hussain, T., Ishtiaq, M., Hussain, A. and Sultana, K. (2011). Study of drinking water fungi and its pathogenic effects on human beings from District Bhimber, Azad Kashmir, Pakistan. Pakistan Journal of Botany 43 (5), 2581-2585.

Hussein, H. and Brasel, J. (2001). Toxicity, metabolism and impact of mycotoxins on human and animals. Toxicology 167, 101-134.

James, G. C. and Natalie, S. (2001). Microbiology. A laboratory Manual (ed.) pp 211 - 223

Kanzler, D., Buzina, W., Paulitsch, A., Haas, D., Platzer, S., Marth, E., and Mascher, F. (2008). Occurrence and hygienic relevance of fungi in drinking water. Mycoses, 51(2), 165-169.

Khan, M. S., Ahmad, I., Aqil, F., Owais, M., Shahid, M. and Musarrat, J. (2010). Virulence and pathogenicity of fungal pathogens with special reference to Candida albicans. In: Combating Fungal Infections: Problems and Remedy (I. Ahmad, M. Owais, M. Shahid and F. Aqil, eds). Springer- Verlag, Berlin, Heidelberg, Germany, pp. 21-45.

Korzeniewska, E. (2011). Emission of bacteria and fungi in the air from wastewater treatment plants: a review. Bioscience Reports 1 (3), 393-407.

Lowenberg, U. and Kunzel, T. (1992). Investigations on the hydrology of the Lower Cross River, Nigeria. Animal Research and Development, Vol. 35. pp. 73-85. Institute for Scientific Cooperation, Tubingen.

Magwaza, N., Nxumalo, E. N., Mamba, B. B. and Msagati, T. A. M. (2004). The occurrence and diversity of waterborne fungi in African aquatic systems: their impact on water quality and human health. International Journal of Environmental Research and Public Health $14(6), 546$. 
Máiz, L., Nieto, R., Cantón, R., de la Pedrosa, E. G. G. and Martinez-García, M. Á. (2018). Fungi in bronchiectasis: aconcise review. International Journal of Molecular Sciences 19 (1), $1-13$.

Ogunkoya, O. O. (2012). All Rivers run into the sea; yet the sea is not full. Inaugural lecture series, 256. Obafemi Awolowo University, Ile-Ife, Nigeria.

Omar, H. E. M. 2013. Mycotoxins-induced oxidative stress and disease. Chapter 3. In: Mycotoxin and Food Safety in Developing Countries. InTech Open Science, Rjeka, Croatia, pp. 63-92.

Paterson, R. R. M. and Lima, N. (2008). Fungal Growth and Mycotoxin Production in Drinking Water. IBB - Biological Engineering Centre, University of Minho, Braga, Portugal.

Pereira, V. J., Fernandes, D., Carvalho, G., Benoliel, M. J., San Romao, M. V. and BarretoCrespo, M. T. (2010). Assessment of the presence and dynamics of fungi in drinking water sources using cultural and molecular methods. Water Research 44, 4850-4859.

Pereira, V. J., Marques, R., Marques, M., Benoliel, M. J., and Crespo, M. B. (2013). Free chlorine inactivation of fungi in drinking water sources. Water research, 47(2), 517-523

Pfaller, M. A. and Diekema, D. J. (2004). Rare and emerging opportunistic fungal pathogens: concern for resistance beyond Candida albicans and Aspergillus mini review. Journal of Clinical Microbiology 42 (10), 4419-4431.

Pitt, J. I. (2000). Toxigenic fungi and mycotoxins. British Medical Bulletin 56 (1), 184-192.

Pitt, J. I., Bacilico, J. C., Abarca, M. L. and Lopez, C. (2000). Mycotoxins and toxigenic fungi. Medical Mycology 38 (1), 41-46.

Pitt, J. I., Bacilico, J. C., Abarca, M. L. and Lopez, C. (2000). Mycotoxins and toxigenic fungi. Medical Mycology 38 (1), 41-46.

Sammon, N. B., Harrower, K. M., Fabbro, L. D., and Reed, R. H. (2011). Three potential sources of microfungi in a treated municipal water supply system in sub-tropical Australia. International journal of environmental research and public health, 8(3): 713-732.

Siqueira, V. M. (2011). Characterizing Filamentous Fungal Biofilm in Drinking Water Distribution Systems Using Microscopic and Molecular Techniques. PhD thesis, Universidade do Minho, Portugal.

Smyth, A.J. and Montgomery, R.F. (1962). Soils and Land Use in Central Western Nigeria. Soils and Land Use in Central Western Nigeria.

Sonigo, P., De Toni, A. and Reilly, K. (2011). A Review of Fungi in Drinking Water and the Implications for Human Health. Final report WD 0906. Bio Intelligence Service, Paris, France 
Sonigo, P., De Toni, A. and Reilly, K. (2011). A Review of Fungi in Drinking Water and the Implications for Human Health. Final report WD 0906. Bio Intelligence Service, Paris, France.

Sulaiman, I., Jacobs, E., Simpson, S. and Kerdahi, K. (2014). Molecular identification of isolated fungi from unopened containers of Greek yogurt by DNA sequencing of internal transcribed spacer region. Pathogens 3 (3), 499-509.

Volk, T. J. (2013). Fungi. Encyclopedia of Biodiversity 3, 624-640.

Wurzbacher C., Kerr J., Grossart H.-P. (2011). Aquatic fungi. In: Grillo O., Venora G., (Editors). The Dynamical Processes of Biodiversity: Case Studies of Evolution and Spatial Distribution. 1st ed. Volume 1. InTech; Rijeka, Croatia: 2011. pp. 227-258.

Wurzbacher, C., Kerr, J. and Grossart, H.-P. (1998). Aquatic fungi. In: The Dynamical Processes of Biodiversity - Case Studies of Evolution and Spatial Distribution (O. Grillo \& G. Venora, eds). InTech, Rjeka, Croatia, pp. 227-258.

Zain, M. E. (2011). Impact of mycotoxins on humans and animals. Journal of Saudi Chemical Society 15 (2), 129-144. 